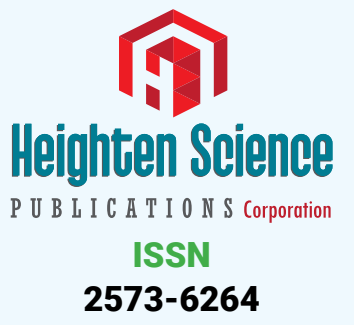

2573-6264
Research Article

\section{Rehabilitation of proximal humerus fractures: An environmental scan of Canadian physiotherapy practice patterns}

\author{
Lowell L Kwan ${ }^{1}$ and Norma J Maclntyre ${ }^{2 *}$ \\ 'Physiotherapist, School of Rehabilitation Science, McMaster University, IAHS-403, 1400 Main \\ St. West, Hamilton, Canada \\ ${ }^{2}$ Associate Professor, School of Rehabilitation Science, McMaster University, IAHS-403, 1400 \\ Main St. West, Hamilton, Canada
}

\title{
Abstract
}

Submitted: 19 August 2017

Approved: 19 September 2017

Published: 20 September 2017

Copyright: @ 2017 Kwan LL, et al. This is an open access article distributed under the Creative Commons Attribution License, which permits unrestricted use, distribution, and reproduction in any medium, provided the original work is properly cited.

Keywords: Shoulder fractures; Physical therapy; Rehabilitation; Questionnaire

Abbreviations: AAROM: Active Assisted Range Of Motion; AROM: Active Range of Motion; GE Strength: Gravity Eliminated Muscle Strengthening; GHJ Mob: Glenohumeral Joint Accessory Movement Mobilization Techniques; PHF(S): Proximal Humerus Fracture(s); PR: Strength: Progressive Resistive Muscle Strengthening; PROM: Passive Range Of Motion/ Pendulum Exercises

\begin{abstract}
Maclntyre, Associate Professor, School of Rehabilitation Science, McMaster University, IAHS-403, 1400 Main St. West, Hamilton, Canada, Tel: 1-905-525-9140, Fax: 1-905-524-0069; Email: macint@mcmaster.ca

*Address for Correspondence: Norma J
\end{abstract}

Background: Proximal humerus fractures (PHFs) are common injuries particularly in older adults. Evidencebased protocols for PHF rehabilitation are lacking and physiotherapists use a variety of interventions.

Purpose: To determine practice patterns and perceptions of physiotherapists who treat adults with PHF in Ontario, Canada.

Method: A paper and pencil survey asking about respondent demographics and management of Neer Group 1 (minimally/nondisplaced) and complex (displaced 3- and 4-part) PHF was mailed to 875 randomly selected physiotherapists who were registered with the College of Physiotherapists of Ontario in 2013/2014 and working in practice areas likely to be accessed by adults with PHF.

Results: The response rate was low (10\%); 83 physiotherapists completed the survey - $80 \%$ had experience managing PHF. Respondents treated 1-5 individuals with PHF annually; more treated Neer Group 1 PHF (89\%) than complex PHF (68\%). Most individuals with PHF were older than 60 years (64\%), female (76\%) and accessed physiotherapy through a doctor's referral (91\%) more than 1 month post injury (33\%).

Main findings: Physiotherapists manage PHF using multi-component interventions and a minimum of $76 \%$ include the following elements: education and progression of passive, active assisted, active range of motion exercises and muscle retraining to build coordination and strength. Use of other elements was variable. The main factors influencing the treatment plan were the ability of the individual with PHF to comply, bone quality, and fracture type. Most respondents were unsure that there is sufficient PHF rehabilitation literature to guide treatment.

Conclusions: This environmental scan is the first North American study to document practice patterns and attitudes of physiotherapists providing PHF rehabilitation. Elements used by physiotherapists in Ontario treating small numbers of individuals with Neer Group 1 or complex PHFs each year align well with the limited PHF rehabilitation literature available.

Potential implications: Multi-disciplinary collaborations to design and conduct large, high quality, multi-centre prognostic studies and RCTs that evaluate the effectiveness of key aspects of non-surgical PHF rehabilitation in various patient groups are needed. Meanwhile, consensus guidelines should be developed in the context of regionspecific physiotherapy service models to inform best practice in PHF rehabilitation management.

\section{Introduction}

Proximal humerus fractures (PHFs) are the third most common upper extremity fracture across the lifespan [1]. Population-based studies estimate similar annual incidence rates in North America and Europe (60 and 82 per 100,000 person years, respectively) [1,2]. The incidence of PHF increases with age and $70.5 \%$ occur in women $[1,2]$. In fact, PHF is the second most common upper extremity fracture in adults 65 years of age and older at which point the annual incidence is 253 per 100,000 person years [1]. Based on emergency and inpatient hospital visits only, the annual economic 
burden of PHF in the United States is estimated to be $\$ 133,469$ [1]. PHFs represent a significant health care problem, especially among the aging population.

Most PHF (62\%) are 2-part fractures and 78\% are treated non-surgically [2]. The incidence decreases as the severity increases across the spectrum of PHF [3]. Although displaced 3- and 4-part PHF are less common, rehabilitation is important as few individuals recover their pre-injury level of functional ability and quality of life $[4,5]$. This may result in considerable morbidity and loss of independence among elderly people at risk for poor functional outcomes.

Regardless of the severity of the PHF, physiotherapists play a vital role in the non-surgical and post-surgical management. However, the most recent Cochrane Systematic Review evaluating the effectiveness of interventions for treating PHF in adults was unable to pool outcomes and recommend specific rehabilitation treatment protocols [6]. The only finding in which these authors had sufficient confidence was that surgical repair of displaced PHF does not result in a better outcome compared to non-surgical management and is likely to result in further surgical interventions [6]. Our group conducted a scoping review with the aim to identify strengths and weakness of the literature addressing the PHF rehabilitation theme [7]. We identified a number of ways that the evidence base could be improved and confirm that physiotherapy protocols for PHF rehabilitation are based on current 'good practice' and expert consensus given the gaps in the literature. It follows that variability in the elements included in physiotherapy treatment exists and this variability has been documented to some extent in literature from the United Kingdom [8,9]. To date, only one pilot study evaluating a specific element of a multi-component physiotherapy intervention for PHF has been conducted in North America [10] and the practice patterns among physiotherapists have not been reported. Thus, the purpose of the present study is to determine practice patterns and perceptions of physiotherapists who treat adults with PHF in Ontario.

\section{Materials and Methods}

\section{Survey sample}

Physiotherapists likely to treat PHF were invited to participate in this cross-sectional descriptive survey. To access physiotherapists, we requested email addresses from the College of Physiotherapists of Ontario in 2013 for registrants with the following characteristics: currently employed and working as physiotherapists; has a position of service provider; has a clinical focus of musculoskeletal system or more than one system; provides patient care; practices in general practice or orthopaedics or rheumatology or women's health or geriatric care or sports medicine or emergency or continuing care/long term care; and works with adults or geriatrics or all ages. Business postal mailing addresses were provided for 4,015 registrants matching our criteria and, of these, 3,987 were practicing in Ontario at that time. Using an online survey sample size calculator (http://www.surveysystem.com/sscalc.htm), we determined a sample of 350 is required to have a confidence level of $95 \%$ and confidence interval of 5 . We then increased this value, anticipating a $40 \%$ response rate [11], resulting in a target sample size of 875 .

Between February and March 2014, we mailed a letter of invitation and the paper and pencil survey to the primary place of occupation of 875 physiotherapists, randomly selected from the mailing list provided. The letter informed recipients of the nature and purpose of the study and invited them to participate. Participants were asked to return the completed survey by email, facsimile, or postal mail using the enclosed selfaddressed stamped envelope. The letter of information also advised participants that an electronic version (fillable PDF) of the survey could be requested if preferred. All survey responses were anonymised. 


\section{Study tool}

We developed a survey to describe current physiotherapy practices in the management of PHF in Ontario based on the current literature. Two studies were most influential: one evaluated physiotherapy management of Neer Group 1 (minimally or nondisplaced with 1 to 4 fracture lines) PHF [9]; one evaluated orthopaedic surgeons' treatment preferences for management of complex PHF (displaced 3- and 4-part) [12]. Four physiotherapists, with expertise in PHF rehabilitation and not working in Ontario at the time of the study, piloted the survey and provided feedback regarding ease of interpretation and relevance of the content. The final version of the survey assessed demographics of participants, characteristics of individuals with PHF, treatment of Neer Group 1 and complex PHFs, factors affecting treatment and opinions on the state of PHF rehabilitation literature. (The survey is shown in Appendix I). Rehabilitation of both Neer Group 1 PHFs and complex PHFs was investigated as the former is most common and the latter is most difficult to treat whether acute management involves surgery or not [12].

\section{Ethics}

The study protocol and study tool received ethics approval from the Hamilton Integrated Research Ethics Board (HiREB-13-817-S). Submission of the completed survey served as informed consent.

\section{Data analysis}

Responses to the survey questions were manually inputted and summarized with Microsoft Excel. Missing data were not imputed. Response rates were calculated for each question by dividing the number of responses by the total number of questionnaires returned without missing data and multiplying by 100 to obtain the percentage. Overall response rate was calculated as recommended by Shih and Xitao [11]. Descriptive statistics were generated for all categorical variables and raw counts and proportions were used to describe demographical information. We used thematic analysis for the 3 optional open-ended questions where an idea must occur at least 3 times to be considered a theme. For these questions, consensus was achieved by both authors (LLK, NJM) in an iterative manner. A conservative estimate of individuals with PHF over the age of 60 years was calculated by dividing the number of individuals over the age of 60 years with PHF by the total number of individuals with PHF. The number of individuals over the age of 60 years was determined as the sum of the raw counts provided by the respondent. If a respondent provided a percentage or range, the lowest possible value was utilized in our calculations. The total number of individuals with PHF was the sum of the highest value in each range selected by the respondent. Statistical tests of inference were not performed due to the descriptive nature of the study.

\section{Results}

Twenty-one of 875 surveys (2\%) could not be delivered and were returned to the research team due to change in address $(n=5)$, change in employment $(n=2)$, clinic closure $(n=1)$ or unknown reasons $(n=13)$. A total of 83 physiotherapists returned their questionnaires (response rate $10 \%)$. These surveys were returned by email $(n=3$, $4 \%)$, facsimile $(n=44,53 \%)$ and postal mail $(n=36,43 \%)$.

\section{Characteristics of respondents and individuals with PHF}

Table 1 summarizes the demographical information provided by the 83 respondents. Seventeen respondents never treat PHF and completed only Part 1 (background information) of the survey.

Questions regarding the characteristics of clients with PHF were answered by 66 respondents (80\%). Most of the people with PHF treated by the respondents (64\%) 


\begin{tabular}{|c|c|}
\hline Characteristics & Number of respondents (\%) \\
\hline \multicolumn{2}{|l|}{ Gender } \\
\hline Female/ Male & $63(76 \%) / 20(24 \%)$ \\
\hline \multicolumn{2}{|l|}{ Age } \\
\hline $21-30$ years & $30(36 \%)$ \\
\hline $31-40$ years & $27(33 \%)$ \\
\hline $41-50$ years & $15(18 \%)$ \\
\hline $51-60$ years & $9(11 \%)$ \\
\hline$>60$ years & $2(2 \%)$ \\
\hline \multicolumn{2}{|l|}{ Years in practice } \\
\hline$<5$ years & $29(35 \%)$ \\
\hline $5-10$ years & $18(22 \%)$ \\
\hline $11-15$ years & $12(14 \%)$ \\
\hline $16-20$ years & $4(5 \%)$ \\
\hline 21-25 years & $14(17 \%)$ \\
\hline$>25$ years & $6(7 \%)$ \\
\hline \multicolumn{2}{|c|}{ Clients with PHF treated per year on average } \\
\hline 0 clients & $17(20 \%)$ \\
\hline $1-5$ clients & $49(59 \%)$ \\
\hline 6-10 clients & $10(12 \%)$ \\
\hline $11-15$ clients & $3(4 \%)$ \\
\hline $16-20$ clients & $1(1 \%)$ \\
\hline$>25$ clients & $3(4 \%)$ \\
\hline
\end{tabular}

were over the age of 60 years. Of the 65 respondents who described patient access to physiotherapy, 59 (90.8\%) reported that this occurs primarily by doctor's referral. Very few respondents indicated that most individuals with PHF access physiotherapy services as a result of direct access/self-referral $(n=3,4.6 \%)$ or other means $(n=3$, 4.6\%). The time period between injury and attending the first physiotherapy session varied: less than 1 week $(n=9,14.1 \%), 1$ week $(n=7,10.9 \%), 2$ weeks $(n=15,23 \%), 3$ weeks $(n=7,11 \%), 4$ weeks $(n=5,7.8 \%)$ and more than 4 weeks $(n=21,33 \%)$.

Of the 66 respondents who treated PHF, 59 (89\%) had treated Neer Group 1 PHF, $45(68 \%)$ had treated complex PHF and 42 (64\%) had treated both types of PHF. Two respondents $(3 \%)$ reported treating other types of PHFs that were not addressed in the survey.

\section{Management of Neer Group 1 PHF}

Figure 1 summarizes the confidence of respondents in managing Neer Group 1 PHF. Five respondents who indicated that they have never treated a client with a Neer Group 1 PHF report that they are not at all confident $(n=1)$ or somewhat confident $(n=4)$.

Of the 57 respondents who answered questions about the use of immobilization in adults with Neer Group 1 PHF, 38 respondents (67\%) indicated that immobilization was used after injury and 5 respondents (9\%) indicated that immobilization was not used. Fourteen respondents (24\%) indicated that "sometimes" the PHF is immobilized and $50 \%$ indicated that a sling was used. Figure 2 summarizes the immobilization periods reported for adults with Neer Group 1 PHF (closed bars) which vary from less than 1 week to 8 weeks or longer.

The question regarding the number of physiotherapy sessions provided, on average, for adults with Neer Group 1PHF was answered by 51 respondents. Most provide 11 to 15 sessions $(n=23,39 \%)$ or 6 to 10 sessions $(n=15,25 \%)$. A few provide less than 5 sessions $(n=7,12 \%), 16$ to 20 sessions $(n=7,12 \%)$ or more than 20 sessions $(n=7$, $12 \%)$.

Figure 3 summarizes the different physiotherapy elements included in the multicomponent treatment of adults with Neer Group 1 PHF. The 59 respondents identified 


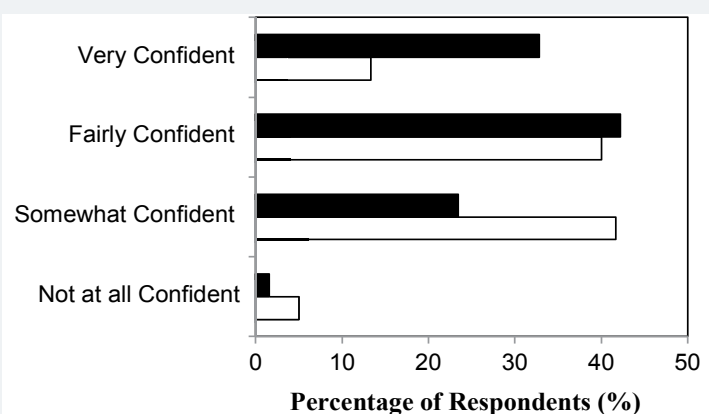

Figure 1: Confidence in managing Neer Group 1 (closed bars, $n=64$ respondents) and complex (open bars, $n=63$ respondents) proximal humerus fractures.

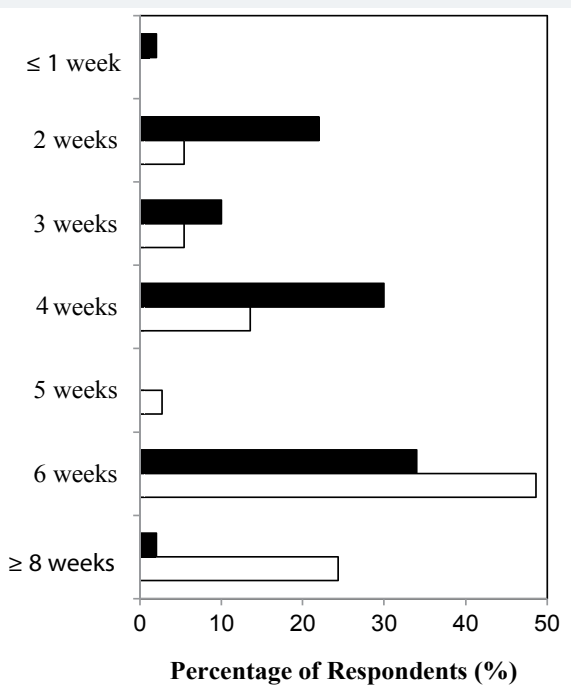

Figure 2: Duration of sling immobilization for Neer Group 1 (closed bars, $n=50$ respondents) and complex (open bars, $\mathrm{n}=37$ respondents) proximal humerus fractures.

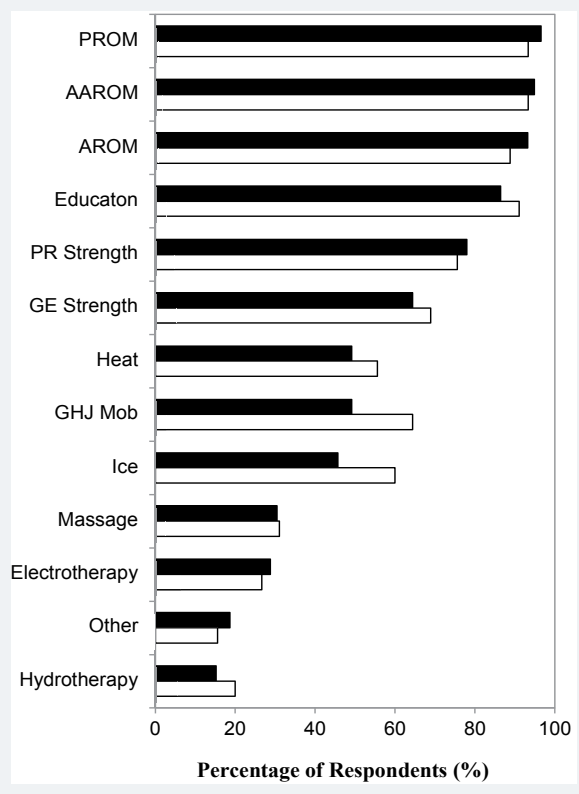

Figure 3: The percentage of respondents reporting use of specific elements in multi-component physiotherapy interventions for Neer Group 1 (closed bars, $n=59$ respondents) and complex (open bars, $n=45$ respondents) proximal humerus fractures. PROM: passive range of motion/pendulum exercises; AAROM: active assisted range of motion; AROM: active range of motion; PR strength: progressive resistive muscle strengthening; GE Strength: gravity eliminated muscle strengthening; GHJ Mob: glenohumeral joint accessory movement mobilization techniques. 
a total of 443 treatment elements for an average of 7.63 (SD=2.24) elements per respondent. Common treatments reported by 11 respondents under 'other' included acupuncture/dry needling $(n=3)$ and general fitness, posture and functional mobility exercises $(n=3)$.

Twenty-five respondents elaborated on the details of their treatment plan. Common themes include progression of the multi-component treatment $(n=18)$, early stage treatment $(n=17)$, treatment of another body part other than the shoulder $(n=6)$, differences in practice setting/service delivery $(n=6)$ and surgeon input $(n=4)$.

\section{Management of complex PHF}

Figure 1 summarizes the confidence of respondents in managing complex PHF regardless of prior experience with this fracture. The 3 respondents who were not at all confident had never treated an adult with a complex PHF. The 12 other respondents who had not treated clients with this type of PHF previously were somewhat confident $(n=8)$ or fairly confident $(n=4)$.

Of 45 respondents who had treated adults with complex PHFs, the majority of their clients were immobilized after injury $(n=39,87 \%)$. In contrast, 1 respondent $(2 \%)$ indicated that immobilization was not used and 5 respondents (11\%) indicated that immobilization was used sometimes. Figure 2 summarizes the immobilization periods reported for adults with complex PHF (open bars) which vary from less than 1 week to 8 weeks or longer.

The question regarding the number of physiotherapy sessions provided, on average, for adults with a complex PHF was answered by 44 respondents. Most provide 16 to 20 sessions $(n=14,32 \%)$, more than 20 sessions $(n=11,25 \%)$, or 11 to 15 sessions $(n=10$, $23 \%)$. A few provide less than 5 sessions $(n=5,11 \%)$ or 6 to 10 sessions $(n=4,9 \%)$.

Figure 3 summarizes the different physiotherapy elements included in the multicomponent treatment of adults with a complex PHF. The 45 respondents identified a total of 353 treatment elements for an average of 7.9 (SD=2.9) elements per respondent. A variety of treatments were listed under "other" by 7 respondents and the only common theme was acupuncture/dry needling $(n=3)$.

Thirteen respondents elaborated on the details of their treatment plan. Common themes include progression of the multi-component treatment $(n=7)$, early stage treatment $(n=5)$, surgical management factors such as surgeon input and comorbidities $(n=4)$ and differences in practice setting/service delivery $(n=4)$. Figure 4 illustrates the use of surgical interventions prior to starting rehabilitation.

\section{Major factors affecting PHF treatment and the state of the evidence}

Most respondents $(n=64,77 \%)$ completed the last part of the survey addressing factors impacting the treatment plan and opinions on the PHF rehabilitation literature.

Figure 5 illustrates the most influential factors guiding PHF treatment. The most frequently reported factors of primary importance are the ability of the individual with PHF to comply with treatment $(n=17,29 \%)$, the fracture type $(n=15,20 \%)$ and "other" factors $(n=14,23 \%)$. Of the 14 respondents who selected "other", 11 specified input from orthopaedic surgeon. The most frequently reported factors ranked second in importance are the ability of the individual with PHF to comply with treatment $(n=23$, $36 \%)$, bone quality $(n=12,20 \%)$ and fracture type $(n=9,14 \%)$. The most frequently reported factors ranked third in importance are bone quality $(n=15,24 \%)$, the ability of the individual with PHF to comply with treatment $(n=14,22 \%)$ and clinical expertise $(n=13,21 \%)$. A total of 167 factors that affect PHF treatment were reported and the 3 most influential factors overall were the ability of the individual with PHF to comply with treatment $(n=47,28 \%)$, bone quality $(n=32,19 \%)$, and fracture type $(n=30,18 \%)$. 


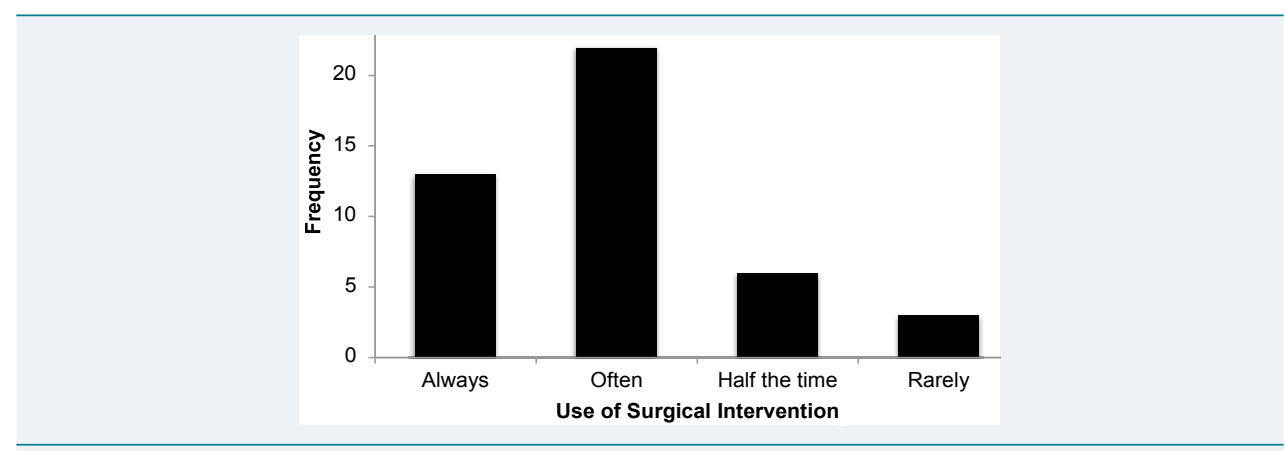

Figure 4: The frequency of reported use of surgical interventions prior to the initiation of rehabilitation of complex proximal humerus fractures ( $n=44$ respondents).

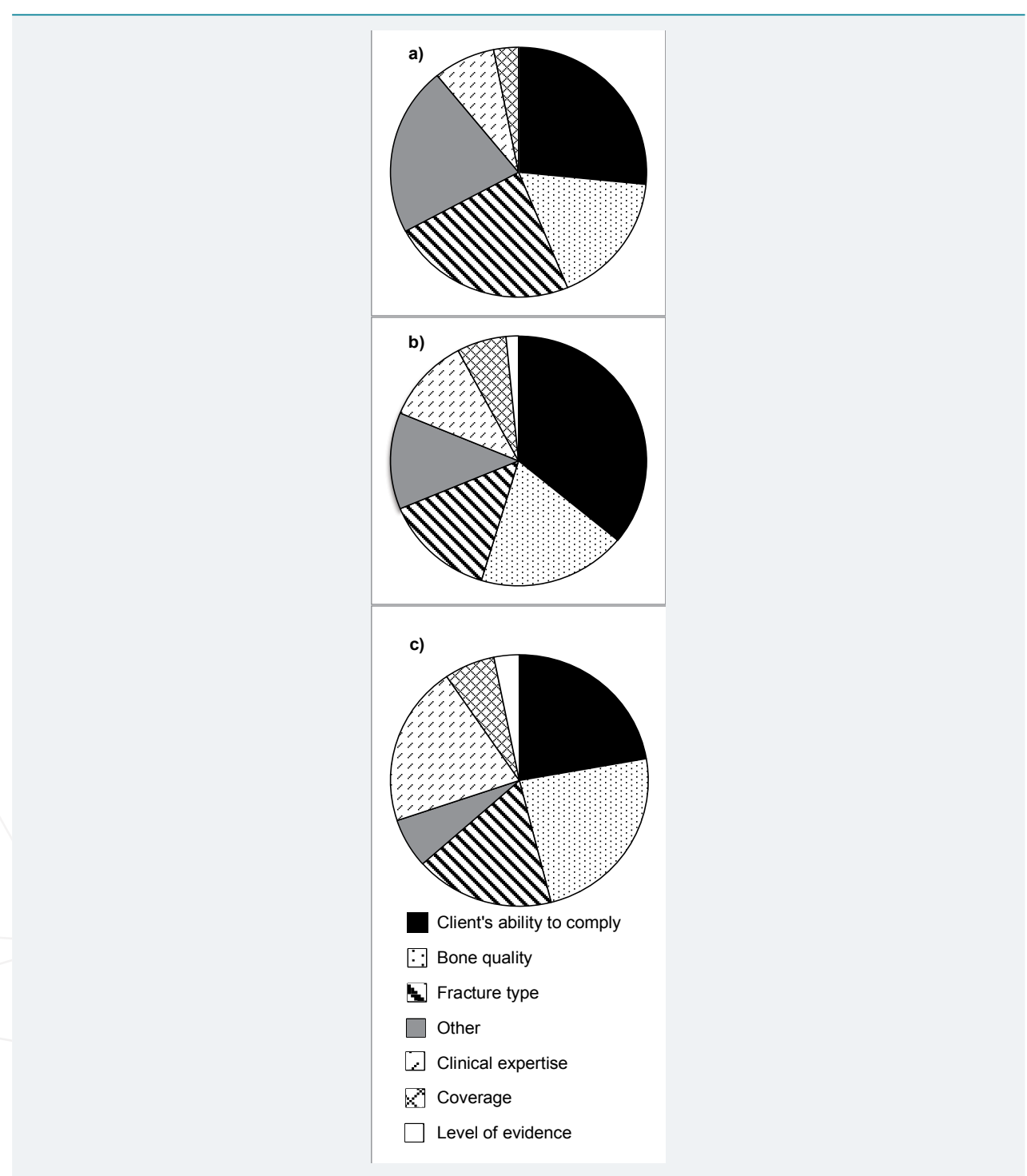

Figure 5: Major factors influencing the treatment provided by the respondents ranked as a) primary consideration $(n=64), b)$ secondary consideration $(n=64)$ and c) tertiary consideration $(n=63)$.

The majority of respondents $(\mathrm{n}=31$ of $55,56 \%)$ are unsure if there is enough literature to guide their management of clients with PHF. Some believe there is not enough literature to guide PHF management $(n=17,31 \%)$ and a few $(n=7,13 \%)$ believe there is enough literature to guide treatment. Eighteen respondents elaborated on research that would better guide their treatment of PHF. The most prominent themes were to investigate the impact of various treatments on stages of fracture/bone healing $(n=6)$, and to develop best practice guidelines $(n=6)$. Another research theme 
expressed by 4 respondents was to investigate the differences in treatment protocols in current use (eg. among various physicians and practice settings). Additionally, 3 respondents indicated an interest in evaluating PHF treatment in different populations. However, one of these respondents commented on the difficulties surrounding PHF rehabilitation research due to the multiple types of PHF, variable characteristics of the adults with PHF, the multiple components of surgical and non-surgical interventions to consider and the challenge of recruiting a sufficient number of participants.

\section{Discussion}

The aim of the present study was to determine practice patterns and perceptions of physiotherapists who treat adults with PHF in Ontario, Canada by conducting a postal pencil and paper survey. Individuals with PHFs are typically women over the age of 60 years, access physiotherapy by doctor's referral, and attend the first physiotherapy visit more than a month post-injury. Most physiotherapists treated 1 to 5 individuals with PHF annually; more had treated Neer Group 1 PHF (89\%) than complex PHF (68\%). Although most of the latter respondents treated both types of PHF (64\%), confidence in treating the Neer Group 1 PHF was higher. Most PHFs are immobilized however variations existed in the immobilization periods, elements included in the multi-component treatment and average number of sessions. Most individuals with complex PHF received physiotherapy post-operatively. The main factors influencing the treatment plan were the ability of the individual with PHF to comply, bone quality, and fracture type. This study provides the first environmental scan of practice patterns and perceptions of physiotherapists providing PHF rehabilitation in North America.

The respondents of the survey are representative of physiotherapists practicing across Canada based on age and gender [13]. Our findings can be compared to a similar study assessing the physiotherapy management of Neer Group 1 fractures in hospital-based orthopedic and trauma centres in the United Kingdom in 2002 [9]. Comparatively, individuals with PHF in Ontario access physiotherapy services later and immobilization of the injured shoulder occurs more frequently and for longer periods of time. These differences may be explained by the various practice settings in which the physiotherapists who responded to our survey work. Alternatively, this contrast may reflect differences in surgical interventions used and rehabilitation protocols prescribed by orthopaedic surgeons in Ontario. Based on the number of adults with PHF treated each year, it is clear that few respondents specialize in the rehabilitation of upper limb fractures. The stage along the care pathway at which individuals with PHF accessed physiotherapy services provided by the respondents is unknown and the variability in time since injury may reflect the different clinical settings (eg., acute versus outpatient versus private clinic). A possible explanation for the difference in immobilization periods may arise from the lack of definitive evidence-based immobilization protocols. A scoping review of the PHF literature identified 8 unique small studies investigating the timing of physiotherapy in individuals with Neer Group 1 and complex PHFs using various study designs [7]. At the group level, it appears that healing is not delayed by early mobilization ( 0 weeks) and it appears that joint and soft tissue extensibility is not decreased significantly by later mobilization ( 3 to 6 weeks) over the long-term [7]. Factors such as the client's pain and communication with the physician with respect to fracture characteristics, interventions used, and evidence of clinical healing guide practice currently. The adults with complex PHF treated by the survey respondents were most often seen post-operatively. Current evidence suggests that adults with displaced 1-, 2-, 3- and 4-part PHF treated surgically do not have better functional outcomes compared with those treated non-surgically [6]. Further research is required to determine if the frequency of surgical interventions for this patient group has changed over time, the types of surgical interventions differ from those investigated in the literature, and adults with complex PHF managed non-surgically are referred to physiotherapy for PHF rehabilitation routinely in Ontario. 
The multi-component nature of the physiotherapy treatment and the variability in the specific elements used by our respondents are similar to those reported in the literature. Specific physiotherapy elements are selected and progressed according to the abilities and needs of the individual in order to restore functional independence. The progression of exercises to regain mobility, normal neuromuscular control, and functional muscle strength of the shoulder girdle may be augmented by other elements. Manual joint mobilizations may be helpful in the early phase of rehabilitation if normal scapulohumeral rhythm is not achieved or added in the intermediate phase if stiffness persists [7]. The use of electrotherapy is not recommended with the exception of transcutaneous electrical nerve stimulation [8] or perhaps dry needling (acupuncture) to active trigger points in shoulder girdle muscles during the first treatment session [14]. Our survey respondents reported the use of elements that aligns well with the limited guidance available in the current PHF rehabilitation literature [7-9,14-16].

Education is a prominent element in physiotherapy treatment of adults with Neer Group 1 and complex PHF in Ontario. Although this survey did not capture the characteristics of the education provided by physiotherapists, the results of the survey support the importance of this element. Home exercise programs are the most consistent form of education utilized in the current PHF rehabilitation literature (see Table 1 in [7]).Two randomized controlled trials showed that home exercise programs introduced and progressed over very few physiotherapy sessions and more frequent physiotherapy visits, are equally effective in regaining functional ability in individuals with Neer Group 1 PHF $[17,18]$. Our survey did not provide information regarding how many adults with Neer Group 1 PHF were managed successfully with education and infrequent monitoring of a home exercise program over the short term. Other elements of education include fracture education, advice on pain management, use of normal movement patterns, relaxation techniques, and self-care [9]. Accordingly, this pedagogic role may build rapport with individuals with PHF resulting in increased compliance with treatment. The importance of tailoring the education and advice to the needs of the individual can be appreciated given that the major factor guiding the physiotherapy intervention plan was the ability of the individual with PHF to comply with treatment. Consequently, further research should focus on optimizing the process, content and mode of delivery of the education and advice and home exercise instructions provided by physiotherapists across the early, intermediate, and later phases of PHF rehabilitation tailored according to the individual's risk profile for functional decline.

The relationship between orthopaedic surgeons and physiotherapists is an important aspect of PHF rehabilitation. Overall, surgeons' protocols were the third most important primary factor considered in treatment planning following the ability of the individual to comply with treatment and fracture type. When respondents elaborated on their treatment plans, an emerging theme was the importance of input from the orthopedic surgeon involved in the management. The PHF rehabilitation literature supports collaborative care for individuals with PHF since functional recovery is dependent on a number of factors such as characteristics of the fracture pattern, the person, and healthcare providers, the surgical or non-surgical interventions used, and the context of care such as financial, transportation or geographical barriers to accessing treatment [7]. Input from multiple disciplines and family members may be required to achieve satisfactory functional independence, particularly for older adults with PHF.

Physiotherapists who responded to our survey were aware that current PHF rehabilitation literature is lacking and does not support evidence-based practice recommendations [6,7]. This study provides insight into knowledge gaps identified 
by knowledge users. Physiotherapists identified the need for best practice protocols to guide PHF rehabilitation. A consensus process by an international panel of experts is recommended as an intermediary step until evidence is available from high quality randomized controlled trials. These trials are needed to evaluate specific aspects of multi-component physiotherapy interventions tailored according to the various fracture characteristics, personal demographics and surgical and non-surgical management strategies given the important influences these factors have on PHF rehabilitation.

\section{Strengths and limitations}

The main strength of this study is the unique findings regarding the practice patterns and perceptions of Canadian physiotherapists who are primarily nonspecialists in PHF rehabilitation. Despite the low numbers of adults with PHF seen in most clinical practices, 83 physiotherapists completed and returned the survey. Responses align with the current PHF rehabilitation literature apart from the high rate of surgical management of complex PHF. The main limitation of this study is the low response rate, which increases the risk for non-response bias. This response rate may be explained by the narrow timeframe for submitting the surveys, the limited follow-up reminders, and the reliance on self-identification of physiotherapists who treat PHF in Ontario. Our recruitment strategy aimed to get input from physiotherapists varying in experience with PHF rehabilitation. However, this strategy had limitations. The College of Physiotherapists of Ontario agreed to provide only business mailing addresses of the registrants likely to treat PHF according to criteria described previously. We were unable to access email addresses or collect data regarding practice settings due to the potential that respondents could be identified. We expect that many physiotherapists with expertise in PHF rehabilitation work in orthopaedic trauma centres and the efficiency of delivery to specific individuals within larger institutions is unknown. Given that the postal mail would reach the institution, it is unlikely that it would be returned to sender. Moreover, this recruitment strategy did not capture the numbers and barriers faced by adults with PHF who do not seek physiotherapy treatment. Finally, this environmental scan focused on 2 categories of PHF and did not cover all types or the spectrum of surgical interventions used in the management of adults with PHFs.

\section{Conclusion}

This environmental scan is the first North American study to document practice patterns and perceptions of physiotherapists providing PHF rehabilitation. Responses from physiotherapists in Ontario treating small numbers of adults with Neer Group 1 or complex PHFs each year align with the current PHF rehabilitation literature apart from the duration of immobilization, the use of surgical strategies in the management of complex PHF, and the length of time between the injury and the initial physiotherapy session with the respondents. The survey underlines the need for multi-disciplinary collaborations to design and conduct large, high quality, multi-centre prognostic studies and RCTs that evaluate the effectiveness of key aspects of non-surgical PHF rehabilitation in various patient groups. In the meantime, consensus guidelines should be developed in the context of physiotherapy service delivery in Ontario to inform best practice in PHF rehabilitation management.

\section{Acknowledgements}

The authors thank the survey respondents for participating, the College of Physiotherapists of Ontario for their help in recruitment, and Katie Crockett, Kendra Usunier and Amber Rollack for reviewing the study tool. 


\section{References}

1. Karl JW, Olson PR, Rosenwasser MP. The epidemiology of upper extremity fractures in the United States, 2009. J Orthop Trauma. 2015; 29: 242-244. Ref.: https://goo.gl/MwrqCa

2. Launonen AP, Lepola V, Saranko A, Flinkkila T, Laitinene M, et al. Epidemiology of proximal humerus fractures. Arch Osteoporos. 2015; 10: 209. Ref.: https://goo.gl/u52orr

3. Court-Brown CM, Garg A, McQueen MM. The epidemiology of proximal humerus fractures. Acta Orthop Scand. 2001; 72: 365-371. Ref.: https://goo.gl/EoMbNu

4. Olerud P, Ahrengart L, Ponzer S, Saving J, Tidermark J. Internal fixation versus nonoperative treatment of displaced 3-part proximal humeral fractures in elderly patients: a randomized controlled trial. $J$ Shoulder Elbow Surg. 2011; 20: 747-755. Ref.: https://goo.gl/CfF74Q

5. Olerud P, Ahrengart L, Ponzer S, Saving J, Tidermark J. Hemiarthroplasty versus nonoperative treatment of displaced 4-part proximal humeral fractures in elderly patients: a randomized controlled trial. J Shoulder Elbow Surg. 2011; 20: 1025-1033. Ref.: https://goo.gl/pKvbjJ

6. Handoll HHG, Brorson S. Interventions for treating proximal humeral fractures in adults. Cochrane Database Syst Rev. 2015. Ref.: https://goo.gl/qZMyUr

7. Maclntyre NJ, Kwan LL, Johal H, Lefaivre KA, Sprague S, et al. Rehabilitation of proximal humerus fractures: a scoping review. SM J Trauma Care. 2017; 1:1001. Ref.: https://goo.gl/QGzh33

8. Handoll HHG, Goodchild L, Brealey SD, Hanchard NCA, Jefferson L, et al. Developing, delivering and documenting rehabilitation in a multi-centre randomised controlled surgical trial: experiences from the PROFHER trial. Bone Joint Res. 2014; 3: 335-340. Ref.: https://goo.gl/SDs45Y

9. Hodgson S. Proximal humerus fracture rehabilitation. Clin Orthop Relat Res. 2006; 442: 131-138. Ref.: https://goo.gl/EyfTyE

10. Tousignant M, Giguere AM, Morin M, Pelletier J, Sheehy A, et al. In-home telerehabilitation for proximal humerus fractures: a pilot study. Int $\mathrm{J}$ Telerehabil. 2015; 6: 31-37. Ref.: https://goo.gl/TXvjn7

11. Shih T, Xitao F. Comparing response rates from web and mail surveys: A meta-analysis. Field Methods. 2008; 20: 249-271. Ref.: https://goo.gl/4VudfG

12. Guy P, Slobogean GP, McCormack RG. Treatment preferences for displaced three- and four-part proximal humerus fractures. J Orthop Trauma. 2010; 24: 250-254. Ref.: https://goo.gl/pgpeZx

13. Canadian Institute for Health Information. Physiotherapists in Canada, 2010 National and Jurisdictional Highlights and Profiles. 2017.

14. Arias-Buria JL,Valero-Alcaide R, Cleland JA, Salom-Moreno J, OrtegaSantiago R, et al. Inclusion of trigger point dry needling in a multimodal physiotherapy program for postoperative shoulder pain: a randomized clinical trial. J Manipulative Physiol Ther. 2015; 38: 179-187. Ref.: https://goo.gl/eVWzXN

15. Rubinik $P$, Calmels $P$, Edouard $P$, Genty $M$, Yelnik AP. Care pathways in physical and rehabilitation medicine (PRM): the patient after proximal humeral fracture and shoulder hemi-arthroplasty. Ann Phys Rehabil Med. 2012; 55: 557-564. Ref.: https://goo.gl/NnvCwN

16. Singleton E, Turner R, Gulotta L. Rehabilitation after proximal humerus fracture. Tech Shoulder Elb Surg. 2014; 15: 46-50. Ref.: https://goo.gl/EUDMo3

17. Lundberg BJ, Svenungson-Hartwig E, Wikmark R. Independent exercises versus physiotherapy in nondisplaced proximal humeral fractures. Scand J Rehabil Med. 1979; 11: 133-136. Ref.: https://goo.gl/RZ1AKT

18. Bertoft ES, Lundh I, Ringqvist I. Physiotherapy after fracture of the proximal end of the humerus. Comparison between two methods. Scand J Rehabil Med. 1984; 16: 11-16. Ref.: https://goo.gl/1ypkhG 


\section{APPENDIX I}

PART 1: BACKGROUND QUESTIONS: This part asks 5 questions to provide demographic information.

1) Gender
Female
$\square$ Male
$\square$ Prefer not to disclose

2) Age

21 to 30 years old

31 to 40 years old

$\square 41$ to 50 years old

51 to 60 years old

$\square$ More than 60 years old

3) Number of years in practice

$\square$ Less than 5 years

5 to 10 years

$\square 11$ to 15 years

$\square 16$ to 20 years

$\square 21$ to 25 years

$\square$ More than 25 years

4) On average, how many clients with PHFs do you personally treat in a year?

0 clients per year

$\square 1$ - 5 clients per year

$\square$ - 10 clients per year

$\square 11$ - 15 clients per year

$\square 16$ - 20 clients per year

21 - 25 clients per year

More than 25 clients per year

5) Of those clients with PHF, approximately how many patients are over the age of 60yr?

(n)

If you NEVER treat a client with PHF, please stop here and submit your responses to the first 6 questions. Thank you. 


\section{PART 2: CHARACTERISTICS OF CLIENTS WITH PHF AND PHYSIOTHERAPY MANAGEMENT}

The following two questions ask about the characteristics of your clients with PHF.

6) How do the majority of clients with PHF access your physical therapy services?
Doctor's Referral
$\square$ Direct access/self- referral
$\square$ Don't know
Other:

7) On average, how soon after PHF do your clients have their first physical therapy visit?
$\square 1$ week
$\square 2$ weeks
$\square 3$ weeks
$\square 4$ weeks

$\square$ Less than 1 week

$\square$ More than 4 weeks

\section{PART 3A: NON DISPLACED OR MINIMALLY DISPLACED PHF (NEER GROUP 1) REHABILITATION}

For the following questions, we are interested in the physical therapy treatment of non displaced or minimally displaced PHFs (Neer Group 1). Neer defined Group 1 as a PHF in which no bone segment is displaced more than $1 \mathrm{~cm}$ or angled greater than $45^{\circ}$.

8) How confident are you in managing this type of PHF?

$\square$ Not at all confident

$\square$ Somewhat confident

$\square$ Fairly confident

$\square$ Very confident

If you do not have any experience in treating this type of PHF (Neer group 1), please check here $\square$ and go to the next section.

9) Are clients who have a Neer Group 1 PHF immobilized after injury?
$\square$ Yes
$\square$ No
$\square$ Sometimes

If sometimes, please clarify:

10) If immobilized, on average, how long is the immobilization time?

$\square 1$ weeks or less

$\square 2$ weeks

$\square 3$ weeks

$\square 4$ weeks

$\square 5$ weeks

$\square 6$ weeks

$\square 7$ weeks

$\square 8$ weeks or more 
11) How many physical therapy treatment sessions, on average, do you provide for this type of PHF (Neer group 1)?

$\square$ Less than 5 sessions

6 - 10 sessions

$11-15$ sessions

16-20 sessions

$\square$ More than 20 sessions

12) What treatments do you typically prescribe for this type of fracture? Check all that apply.

$\square$ Active assisted ROM exercises

$\square$ Active ROM exercises

$\square$ Electrotherapy

$\square$ Education

$\square$ Gravity eliminated strengthening exercises

$\square$ Glenohumeral joint mobilization techniques

$\square$ Heat

$\square$ Hydrotherapy

$\square$ Ice

$\square$ Massage

$\square$ Passive ROM/pendulum exercises

$\square$ Progressive resisted strengthening exercises

$\square$ Other:

13) Optional: Please elaborate on your general treatment plan? (e.g. initiation of mobilization, number of treatment sessions, interventions).

\section{PART 3B: COMPLEX PHFS REHABILITATION}

For the following questions, we are interested in the physical therapy treatment of complex PHFs (displaced 3 and 4 parts).

14) How confident are you in managing complex PHF?
$\square$ Not at all confident
$\square$ Somewhat confident
$\square$ Fairly confident

$\square$ Very confident

If you do not have any experience in treating complex PHFs, please check here $\square$ and go to the next section.

15) Do your clients with a complex PHF routinely receive surgical treatment before physical therapy?
$\square$ Always
$\square$ Often
$\square$ Half the time
$\square$ Rarely
$\square$ Never 
16) Are clients with a complex PHF immobilized after injury?
Yes
No
$\square$ Sometimes

If sometime, please clarify:

17) If clients are immobilized following a complex PHF, on average, how long is the immobilization time?
$\square 1$ week or less
$\square 2$ weeks
$\square 3$ weeks
$\square 4$ weeks
$\square 5$ weeks
$\square 6$ weeks
$\square 7$ weeks
$\square 8$ weeks or more

18) How many physical therapy treatment sessions, on average, do you provide for complex PHFs?

$\square$ Less than 5 sessions

$\square 6-10$ sessions

$\square 11-15$ sessions

$\square 16-20$ sessions

$\square$ More than 20 sessions

19) What treatments do you typically prescribe for complex PHFs? Check all that apply.

Active assisted ROM exercises

Active ROM exercises

Electrotherapy

Education

Gravity eliminated strengthening exercises

Glenohumeral joint mobilization techniques

Heat

Hydrotherapy

Ice

Massage

Passive ROM/pendulum exercises

Progressive resisted strengthening exercises

Other:

20) Optional: Please elaborate on your general treatment plan for complex PHF and if it differs when treating clients with non-operative complex PHF versus post-operative treatment (e.g. initiation of mobilization, number of treatment sessions, interventions). 


\section{PART 4: MAJOR FACTORS AFFECTING PHF TREATMENT AND STATE OF THE EVIDENCE}

The first question asks you to rank the three (3) most important factors affecting your treatment of PHF. The last two questions are optional and will guide us in future research on the management of PHFs.

21) From the following list, please rank the top three (3) factors that influence your treatment of a client with PHF ( $1=$ most important factor, 2 = second most important factor, 3 = third most important factor).

__Bone quality

__Client's ability to comply (i.e., comorbidities, functional ability, other)

_Clinical expertise/experience

__Coverage for physical therapy (eg., OHIP, third party payer, personal)

__Fracture type

__Level of evidence

__other (explain):

__Other (explain):

_Other (explain):

22) Optional: Do you believe that there is enough literature to guide your treatment of PHF?

$\square$ Yes

$\square$ No

$\square$ Unsure

23) Optional: What research do you think would be useful to better guide your treatment of PHF?

Thank you for completing this survey! 